\title{
Reversibility in a Plastically Flexible Coordination Polymer Crystal: A High-Pressure Study
}

\author{
Xiaojiao Liu, ${ }^{*+[a]}$ Adam A.L. Michalchuk, ${ }^{*[b]}$ Biswajit Bhattacharya, ${ }^{*[b]}$ Franziska Emmerling, ${ }^{[b]}$ and \\ Colin R. Pulham ${ }^{[a]}$
}

[a] EaStChem School of Chemistry and Centre for Science at Extreme Conditions (CSEC), University of Edinburgh, Edinburgh, UK.

[b] Federal Institute for Materials Research and Testing (BAM), Berlin, Germany.

† These authors contributed equally

Correspondence: xiaojiao.liu@ed.ac.uk; adam.michalchuk@bam.de; biswajit.bhattacharya@bam.de

\begin{abstract}
Single crystals which exhibit mechanical flexibility are promising materials for advanced technological applications. Before such materials can be used, detailed understanding of the mechanisms and structural effects of bending are needed. Coordination polymer single crystal represent a fascinating class of mechanically flexible material; their bending contradicts existing models. Using single crystal X-ray diffraction and microfocus Raman spectroscopy, we study in atomic detail the high-pressure response of the plastically flexible coordination polymer $\left[\mathrm{Zn}(\mu-\mathrm{Cl})_{2}(3,5-\right.$ dichloropyridine $\left.)_{2}\right]_{n}$. In stark contrast to three-point bending, the quasi-hydrostatic compression of the single crystal is completely reversible, even following compression to over $9 \mathrm{GPa}$. A structural phase transition is observed at $c a .5 \mathrm{GPa}$. Ab initio DFT calculations show this transition to result from the pressure-induced softening of low frequency vibrations. This phase transition is not observed during three-point bending. Our combined experimental and theoretical high-pressure investigation propose slight compression at low levels of bending. However, our studies provide the first indication of overall disparate mechanical responses of bulk flexibility and quasi-hydrostatic compression. We suspect this to be a general feature of mechanically plastic materials.
\end{abstract}

The mechanical flexibility exhibited by some single crystals of organic molecules and coordination polymers has attracted widespread attention. ${ }^{1,2}$ Examples are now known to include both elastically (fully reversible) and plastically (irreversible) bendable materials. These fascinating materials offer exceptional opportunities to develop next-generation advanced materials. Applications are already reported for optical wave guide technologies, ${ }^{3}$ as tunable magnets, ${ }^{4}$ and as flexible electronic conductors. ${ }^{5,6}$ In some cases, useful functional properties - such as fluorescence output - are known to vary with the state of bending. ${ }^{7,8}$ Such effects offer significant potential for sensing and display applications.

Models have been proposed to explain the mechanical flexibility of molecular and metal organic crystals. ${ }^{9-11}$ Conceptually, these models are derived from analysis of the intermolecular interactions which are present in the solid state. Plastically flexible crystals typically comprise low energy shear planes, whereas elastically flexible materials often contain relatively weak isotropic intermolecular interactions. To date, these models have proved highly successful for the design of new materials. 
However, an increasing number of mechanically flexible materials are being reported which do not adhere to existing design principles. ${ }^{6,10,12}$ Notable exceptions include the recently discovered class of flexible single crystal coordination polymers (CP). ${ }^{9,13}$ The mechanisms of flexible materials must be better understood before such materials can be selectively designed for targeted, practical applications as advanced functional materials.

The need for further crystallographic detail was recently highlighted in the pioneering work of Reddy and Naumov. ${ }^{11}$ Using microfocus synchrotron X-ray diffraction, deformation of the crystallographic unit cell was measured directly within the deformed region of a bent single crystal. Atomic positions have been since measured in similar experiments, providing unrivaled detail of mechanical flexibility. ${ }^{10,14,15}$ Importantly, X-ray diffraction offers only an averaged picture of the irradiated material; the inhomogeneity of distortion during bending remains an exceptional challenge. ${ }^{16}$ Only a single study has so far attempted to capture this inhomogeneous field by exploring modulated crystallographic distortions in aminoboranes. ${ }^{14}$ Hence, despite impressive efforts, direct approaches to extract atomistic detail of mechanically bent single crystals remains a significant challenge.

It is instead convenient to explore atomistic deformations during mechanical bending indirectly, through models. Early studies proposed that the structural distortions associated with mechanical bending should follow the 'intrinsic' thermal motion of atoms in the crystal lattice. ${ }^{17}$ This notion was recently refuted for the case of an elastically flexible molecular material, $\mathrm{Cu}$ (II) acetylacetonate, ${ }^{18}$ although its validity across a wider array of flexible crystals has not yet been explored. Alternatively, it is widely suggested that mechanical bending results from local compressive and tensile distortions, a hypothesis supported also by the recent microfocus X-ray diffraction studies. ${ }^{10,14}$ Correspondingly, extensive efforts have been devoted to measuring the anisotropic mechanical properties (e.g. Young's modulus and Poisson ratio) of flexible materials. ${ }^{10,11,15,19}$ The mechanical hardness is known to vary across the deformed crystal, hardening at the inner arc. ${ }^{15}$ This strongly suggests an increase of local pressure during bending. These findings have motivated us to explore the effects of hydrostatic compression on the structure of mechanically flexible materials.

Hydrostatic compression to high pressure (HP) is an indispensable tool for elucidating the reactivity and behavior of organic and metal-organic materials. ${ }^{20}$ Through HP X-ray diffraction and vibrational spectroscopy, both the structural and dynamical origins of material mechanical properties are accessible. By systematically exploring structural and dynamical changes at pressure, we expect to elucidate the favored relaxation pathways of flexible materials when exposed to mechanical stress. To the best of our knowledge, no such studies have yet been reported.

As a model system we studied the HP response of a plastically bendable 1D coordination polymer (CP) $\left[\mathrm{Zn}(\mu-\mathrm{Cl})_{2}(3,5 \text {-dichloropyridine })_{2}\right]_{n}(\mathbf{1})$, Figure 1. Isostructural to other mechanically flexible $\mathrm{CPs},{ }^{9,13}$ (1) crystallizes in the tetragonal space group $P \overline{4} b 2$, with covalent $\mathrm{CP}$ chains aligned along the crystallographic $c$-axis. Single crystals of (1) deform plastically across two crystallographic faces when exposed to three-point bending (Figure 1c-d). ${ }^{9}$ The reported vibrational spectra indicated that no structural changes occur within the $\mathrm{CP}$ chains during bending. Instead, the high mosaicity which resulted from bending was ascribed to inter-CP chain distortions. By subjecting (1) to extremes of pressure, we seek to unravel how the bulk plastic flexibility relates to the material atomistic response to pressure. 
(a)

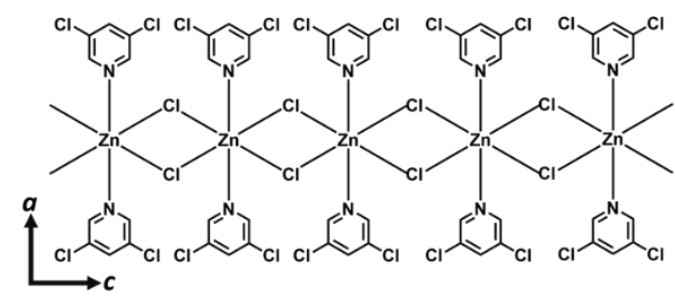

(b)

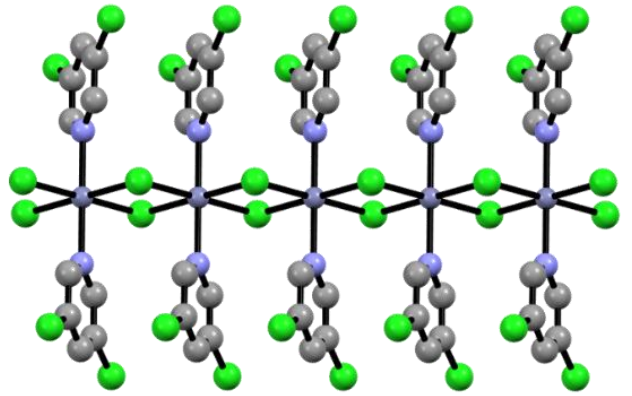

(c)

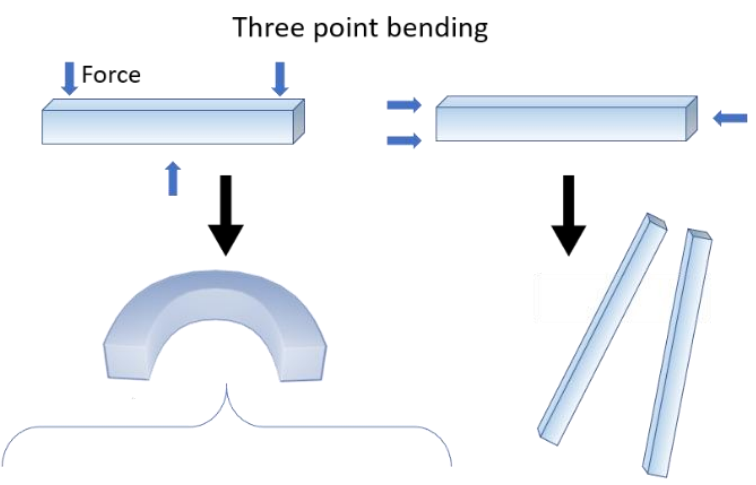

(d)

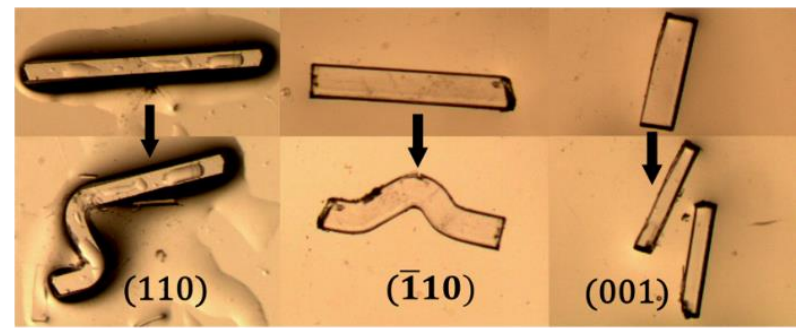

Fig 1: Description of $\left[\mathrm{Zn}(\mu-\mathrm{Cl})_{2}(3,5 \text { - dichloropyridine })_{2}\right]_{n}(\mathbf{1})$, as reported in our previous work. ${ }^{9}$ (a) Schematic representation of the coordination polymer chains of (1). (b) Ball and stick model representation of the coordination polymer chain shown in (a). Atoms are colored as $\mathrm{C}$ - grey; $\mathrm{N}$ - blue; $\mathrm{Cl}$ - green; $\mathrm{Zn}$-dark blue. (c) Schematic representation of three-point bending. Crystal bending is achieved by immobilizing the ends of the crystal on one face and exerting a force in the opposing direction. (d) Microphotographs of plastically bent crystals along the (110) and ( $\overline{1} 10)$ faces, and brittle along the CP chains, i.e., the (001) face.

Our HP X-ray diffraction experiments were conducted using a single crystal of (1) loaded into a Merrill-Bassett diamond anvil cell (DAC) with ruby spheres as the pressure calibrant (see ESI S1). A 1:1 pentane-isopentane mixture was used as pressure-transmitting medium. With increasing pressure, all crystallographic unit cell axes compress monotonically (i.e. smoothly), Figure 2a. Remarkably, the $\mathrm{CP}$ chains ( $c$-axis) compress at a nearly identical rate as the inter-chain voids ( $a$ and $b$ ), thereby yielding a spherical compressibility indicatrix, Figure $2 \mathrm{~b}-\mathrm{c}$. This so-called indicatrix provides a map of the magnitude of compressibility in each direction. ${ }^{21}$ The sphericity of (1) compressibility differs markedly from its variable temperature response, which is highly anisotropic - further details of which will be the subject of a future publication.

Inspection of the atomic structure shows that CP compression occurs as a 'spring', with the Cl-Zn$\mathrm{Cl}$ angles expanding with pressure, Figure $2 \mathrm{~d}$ and $2 \mathrm{f}$. Only minimal changes in the $\mathrm{Cl}-\mathrm{Zn}$ bond lengths is observed, Figure 2e. The HP response of (1) is exceptionally well reproduced by our DFT calculations at PBE-TS level, Figure 2 and ESI S3.2. No significant changes are observed in the electronic structure of the covalent $\mathrm{Zn}-\mathrm{Cl}-\mathrm{Zn}$ chain upon compression, ESI S3.4. 
(a)

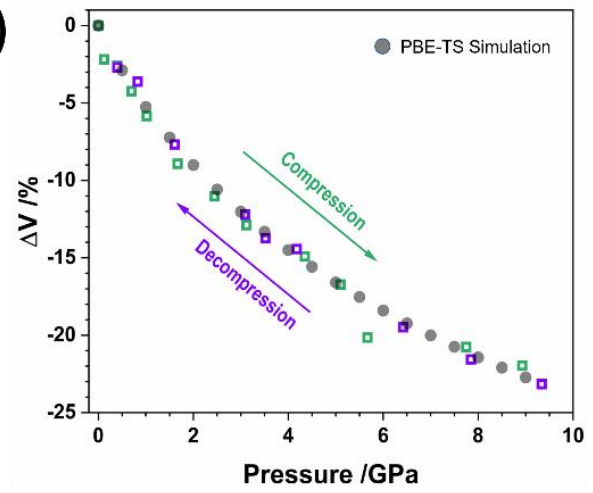

(b)

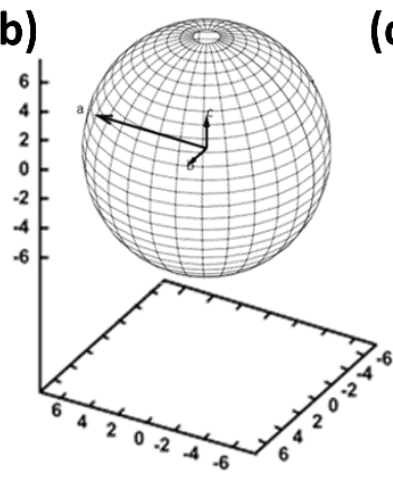

(c)

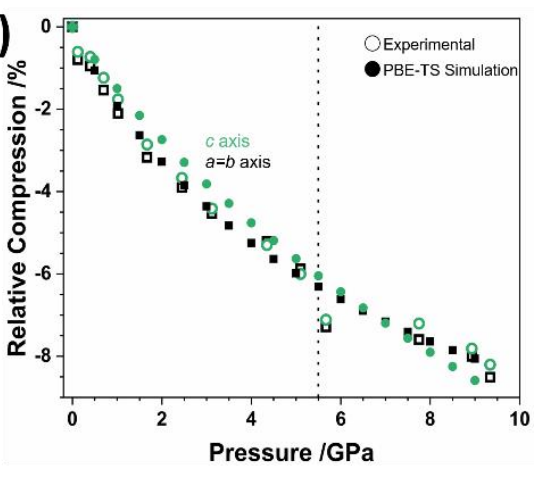

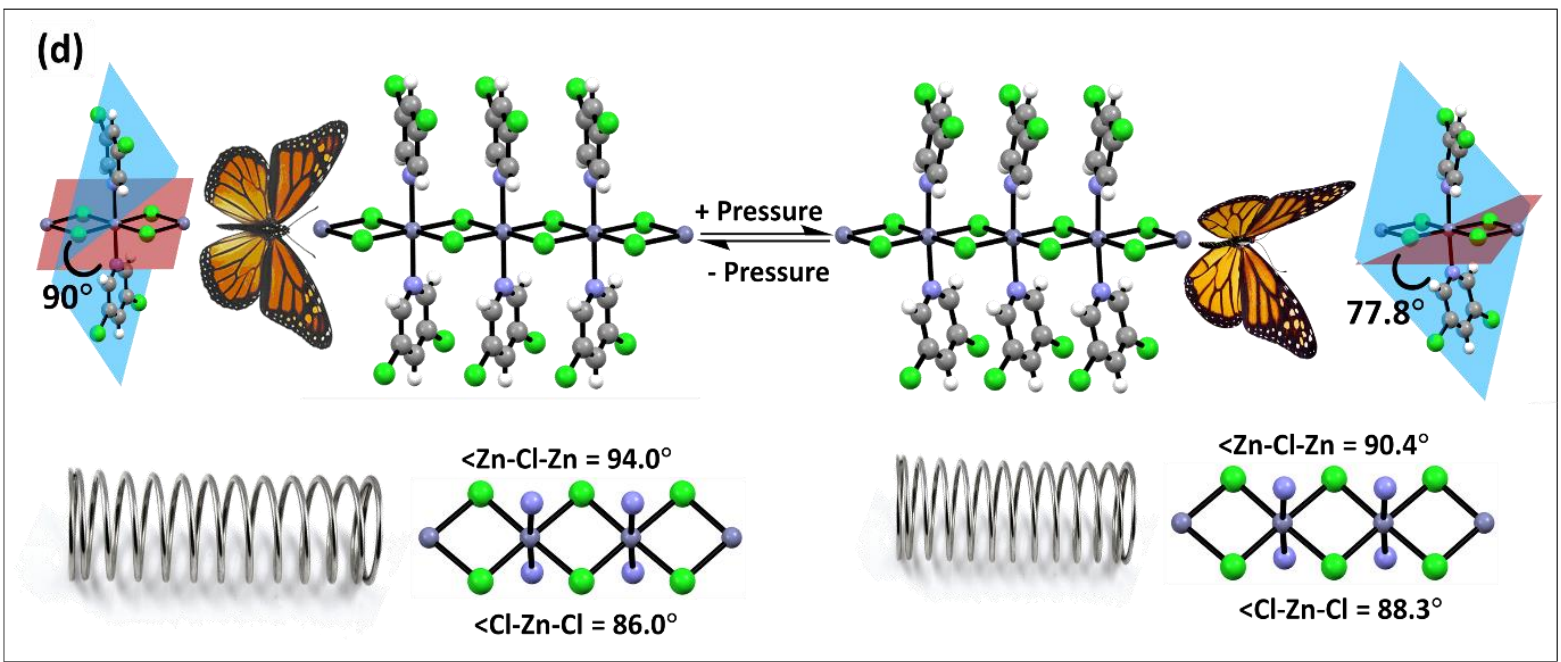

(e)

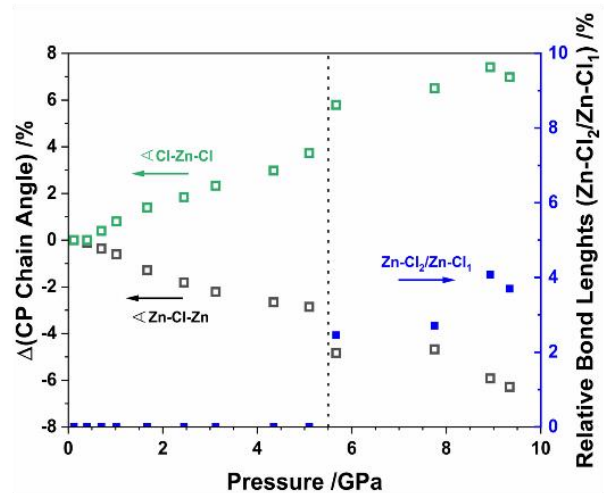

(f)

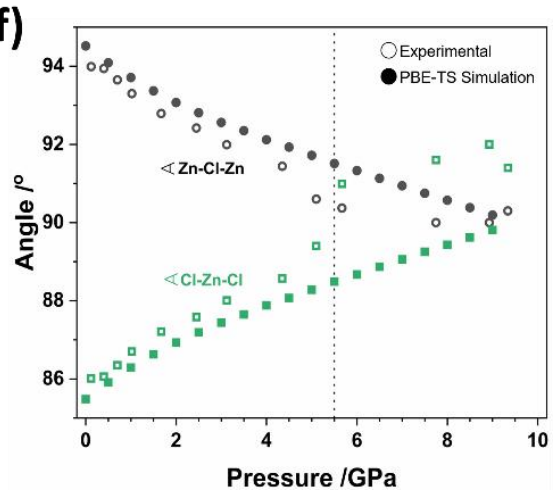

Figure 2: Effects of high pressure on the crystallographic structure of (1). (a) The relative change of the crystallographic unit cell volume during (green) compression and (purple) subsequent decompression. Unit cell volumes obtained by PBE-TS simulations are shown in black. The phase transition point is indicated by a vertical dotted line. (b) The compressibility indicatrix obtained from the pressure derivatives of the unit cell axes of (1) to $9.35 \mathrm{GPa}$. The unit cell axes are indicated by the inset arrows. (c) The relative change of the crystallographic unit cell axes of (1) during compression. Experimental values are shown as open squares (black - a,b axes ; green - $c$ axis) and PBE-TS values are indicated as closed circles (black $a, b$ axes; green - c axis). (d) Effects of the crystallographic phase transition on the CP chain geometry. With increasing pressure, the CP chains are compressed as springs. Across the phase transition a puckering of the chloro-pyridine ligands occurs. This leads to a significant change in angle between the planes of the chloro-pyridine ligands and the CP backbone akin to a butterfly flying motion. (e) Evolution of CP chain geometry with increasing pressure; (green) relative change in $\measuredangle \mathrm{Cl}$ $\mathrm{Zn}-\mathrm{Cl}$ (black) relative change in $\measuredangle \mathrm{Zn}-\mathrm{Cl}-\mathrm{Zn}$, (blue) relative change in $\mathrm{Cl}-\mathrm{Zn}$ bond lengths . (f) Change in $\measuredangle Z n-C l-Z n$ and $\measuredangle \mathrm{Cl}$ $\mathrm{Zn}-\mathrm{Cl}$ angles as a function of pressure, obtained by experiment and PBE-TS simulations. 
Single crystals of (1) exhibit unmistakable plasticity during three-point bending Figure 1c-d..$^{9}$ In stark contrast, the HP response of (1) is fully reversible, even following compression to $9.34 \mathrm{GPa}$. During decompression the crystallographic unit cell relaxes along the same pressure-volume (p-V) path with no detectable hysteresis, Figure 2a-b. The complete reversibility and notable compressibility of the CP chains ( $c$ axis) demonstrate decisively that the bulk mechanical properties exhibited under quasihydrostatic compression do not directly dictate the mechanical properties observed during three-point bending.
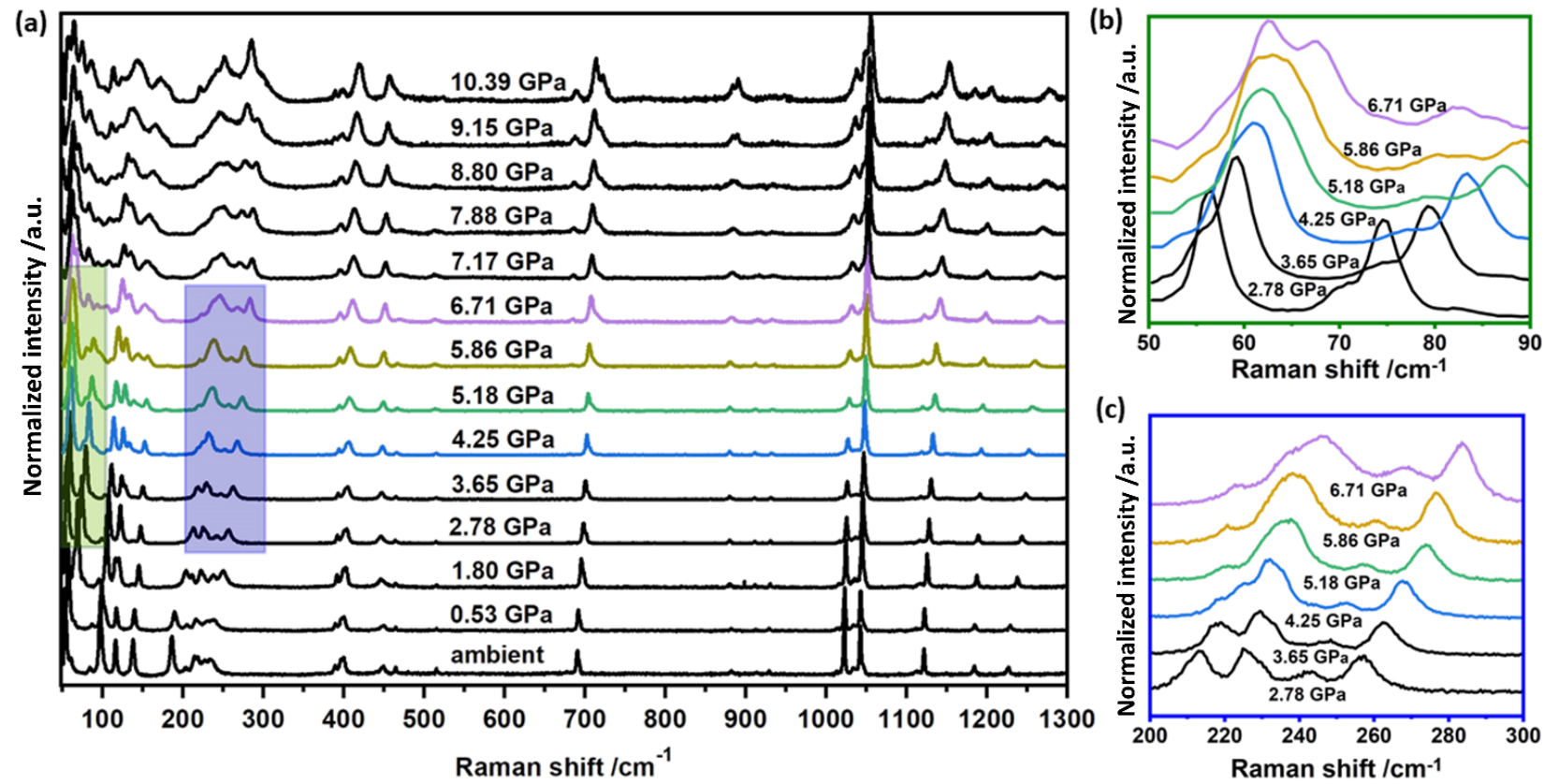

Figure 3: High pressure Raman spectra collected for a polycrystalline sample of (1). (a) Raman spectra collected across complete pressure range; (b-c) magnification of selected regions which exhibit band splitting across the phase transition. Full spectra are given in ESI S5.

Micro-focus Raman spectra provided further detail regarding the HP response of a powdered sample of (1) (see ESI S1). The ambient pressure Raman frequencies are reproduced well by our PBETS approach, thereby allowing band assignments (see ESI S4 and S5). With increasing pressure, significant hardening (i.e. blue shift) of the vibrational modes of (1) was observed, Figure 3a. Consistent with the compression of $\mathrm{Zn}-\mathrm{Cl}$ bonds, the Raman-active (symmetry species $E$ ) $\mathrm{Zn}-\mathrm{Cl}$ stretching modes harden from $c a .93 \mathrm{~cm}^{-1}$ at ambient pressure to $c a 136 \mathrm{~cm}^{-1}$ by $4 \mathrm{GPa}$. In contrast, the Raman bands associated with intramolecular interactions in the dichloro-pyridine ligands (i.e. $>300 \mathrm{~cm}^{-1}$ ) exhibit minimal hardening, Figure 3 and ESI S5. The mode hardening observed under HP conditions contrasts with our previous three-point-bending results, in which no changes were detected in the Raman spectra during bending. ${ }^{9}$ Moreover, compression of (1) did not result in the loss of lattice vibrational bands < $100 \mathrm{~cm}^{-1}$ (Figure 3a and $\mathrm{b}$ and ESI S5) previously noted upon three-point-bending. Instead, the Raman band at $\mathrm{ca} 50 \mathrm{~cm}^{-1}$ (wagging of the pyridyl ligands), which remains unchanged during three-point bending, blue shifts. This shift indicates significant perturbation of the inter-chain interactions upon compression, far beyond the local forces sustained in the crystal during bending. The combined XRD structural and vibrational changes clearly indicate that the HP response of (1) differs significantly from its bending mechanism at ambient pressure. 
The pressure-volume (p-V) curves obtained up to $9.34 \mathrm{GPa}$ exhibit no obvious discontinuities. However, deeper analysis of the HP behaviour reveals the onset of a structural phase transition in (1) between 4-5 GPa, Table 1. Our X-ray diffraction data suggest this to be a continuous transition, reaching only partial conversion by $5.12 \mathrm{GPa}$. By $5.67 \mathrm{GPa}$ the transition appears complete. The phase transition is associated with a loss of symmetry, with the unit cell space group symmetry reducing from $P \overline{4} b 2$ to $P \overline{4}$. The plane formed from the $\mathrm{Zn}-\mathrm{Cl}$ backbone sits orthogonal to the plane formed by either $\mathrm{Zn}$-pyridine in the low-pressure form. In contrast, these same planes jump to internal angles of $\mathrm{ca} 78^{\circ}$ in the high-pressure phase, Figure 2d-f. Hence structurally, this transition is accompanied by puckering of the dichloro-pyridine ligands, akin to a 'butterfly' motion, Figure $2 \mathrm{~d}$.

Table 1: Experimentally determined lattice parameters for (1) before and after the phase transition. Ambient pressure parameters are taken from Ref ${ }^{9}$. The space group (SG) is given in each case

\begin{tabular}{cccc}
\hline & Ambient & $\mathbf{4 . 3 5} \mathbf{~ G P a}$ & 5.67 GPa \\
\hline $\mathbf{S G}$ & $P \overline{4} b 2$ & $P \overline{4} b 2$ & $P \overline{4}$ \\
$\mathbf{a}=\boldsymbol{b} / \AA$ & $13.8212(6)$ & $13.1008(5)$ & $12.8139(14)$ \\
$\boldsymbol{c} / \AA$ & $3.6510(2)$ & $3.4575(2)$ & $3.3912(5)$ \\
$\mathbf{V} / \AA^{3}$ & $697.43(7)$ & 593.414 & 556.822 \\
\hline
\end{tabular}

The structural changes observed across the phase transition break the $\mathrm{C}_{2}$ symmetry of the $\mathrm{Zn}(3,5$ dichloropyridine) ${ }_{2}$ moieties parallel to the $\mathrm{N}-\mathrm{Zn}-\mathrm{N}$ vector (see ESI S3.1). This has the unexpected effect of allowing the $\mathrm{Zn}-\mathrm{Cl}$ bonds above and below the $\mathrm{Zn}$ (3,5-dichloropyridine) ${ }_{2}$ planes to vary independently with pressure, Figure $2 \mathrm{~d}$-f. Whereas before the phase transition all $\mathrm{Zn}-\mathrm{Cl}$ bonds compress by $0.015 \AA . \mathrm{GPa}^{-1}$, they compress asymmetrically after the transition, by $0.017 \AA / \mathrm{GPa}$ and $0.003 \AA / \mathrm{GPa}$ on either side of the pyridyl ligand plane.

Indications of this structural phase transition are also observed in the Raman spectra, Figure 3b-c. This is notable by the onset of splitting in the Raman bands at $c a .60 \mathrm{~cm}^{-1}$ (Figure 3b) and $220 \mathrm{~cm}^{-1}$ (Figure 3c). Splitting of Raman bands is typical of structural phase transitions which result in a decrease of symmetry. According to our PBE-TS simulations, these bands correspond to wagging of the pyridine ligands and an out-of-plane pyridine bending mode, respectively.

The crystallographic unit cell contains the same translational symmetry in both the low- and highpressure phases. Correspondingly, the structural phase transition is dominated by a distortion at the $\Gamma$ point of reciprocal space. Symmetry reduction from point group $D_{2 d} \rightarrow S_{4}$ suggests that a symmetry coordinate of $A_{2}$ character should be responsible. $A_{2}$ modes are not Raman active, and can therefore not be observed in the Raman spectra in Figure 3. Our simulations do, however, reveal a low-energy (25.2 $\mathrm{cm}^{-1}$ ) vibrational mode with atomic displacements that are consistent with the pyridyl ligand 'butterfly' distortion of the structural phase transition. Remarkably, our harmonic simulations show that this $A_{2}$ vibrational band softens (i.e. red shifts) with pressure and becomes dynamically unstable by $4.5 \mathrm{GPa}$, Figure 4a and ESI S4. This is consistent with the experimentally observed pressure for the phase transition.

By incrementally 'freezing' the phonon distortion by factor $\eta$ along the $A_{2}$ eigenvector at each pressure, we mapped out the potential energy surface (PES) of the phase transition, Figure 4b. With increasing pressure, the curvature of the PES decreases, consistent with mode softening. By $4.5 \mathrm{GPa}$ the minimum energy point on the $A_{2}$ PES shifts from $\eta=0$ to $\eta= \pm 4$. Importantly, at the resolution available from our simulations, and within the confines of the harmonic phonon approximation, only a 
single energetic minimum is found at each pressure. This is consistent with a continuous (second order) phase transition in (1). Consistent with experiment, our DFT simulations also show complete reversibility of this vibrationally-driven phase change upon decompression of high-pressure structure (see ESI S3). Hence this structural transition is strongly indicative of the nature of mechanical deformation which occurs in (1). Moreover, the deformation of the CP chain which drives the HP phase transition does not occur during three-point bending, as demonstrated by Raman spectroscopy.
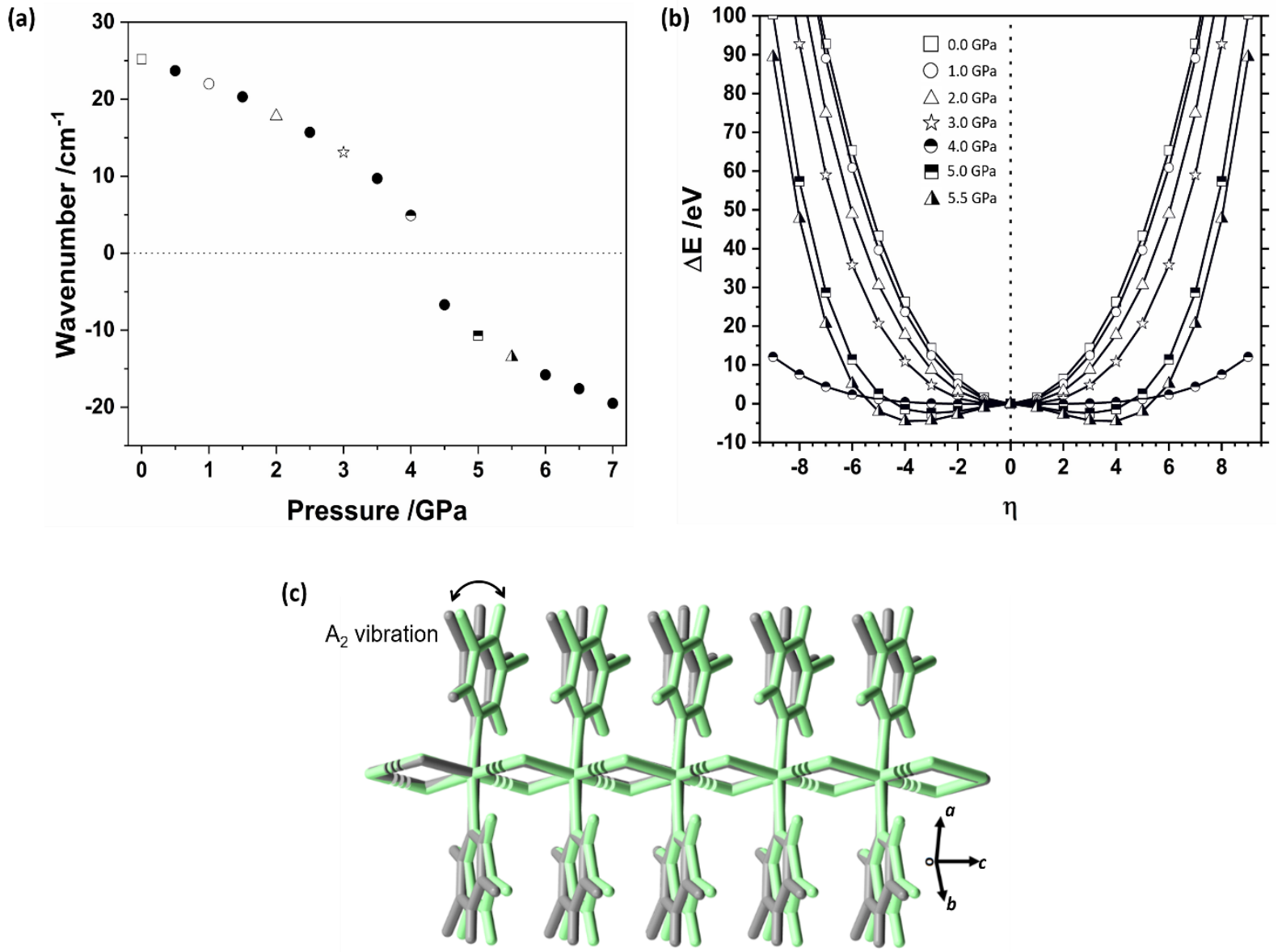

Figure 4: Mode softening of the lowest frequency $A_{2}$ vibrational mode in (1) with pressure. (a) Vibrational frequency of the $A_{2}$ band as a function of pressure. (b) Potential energy surface associated with the lowest frequency $A_{2}$ normal mode in the low pressure phase of (1). Displacements are shown as regular displacements along the $A_{2}$ mode eigenvector away from equilibrium. Note that the curves for $P=5 \mathrm{GPa}$ and $P=5.5 \mathrm{GPa}$ are been multiplied by 10 for clarity. (c) Overlay of the $A_{2}$ normal mode displacement from (grey) $\eta=0$ to (green) $\eta=4$.

Analysis of the PBE-TS eigenvectors obtained from symmetry-constrained DFT calculations shows that the $A_{2}$ vibrational mode is polarised almost exclusively along the crystallographic $c$ axis (ESI S4). Correspondingly, compression of the perpendicular axes is predominantly responsible for the observed phase transition (ESI S6). This is illustrated by simulating the anisotropic compression of (1) along the (100) and (010) axes. In this case, softening of the same $A_{2}$ mode is observed, albeit at significantly lower pressures ( $c a 3 \mathrm{GPa}$ ).

Through an understanding of the high-pressure response of (1) we therefore sought to elucidate the local conditions present within the bent crystals. Our microfocus X-ray diffraction experiments on the bent (i.e. anisotropically stressed) portion of single crystals of (1) show that for crystals bent to 
angles $>90^{\circ}$, only $\mathrm{ca} 1 \%$ compression of the unit cell occurred, predominantly along the $a$ and $b$ crystallographic axes. Our simulations suggest this compression corresponds to local pressures of approximately $0.8 \mathrm{GPa}$, well below the pressures required to achieve the structural phase transition. As bending progresses to angles $>90^{\circ}$, the accumulated stress appears to release, resulting in significant expansion of the crystallographic unit cell. The crystallographic $a$ and $b$ axes expand by $c a 43 \%$, while the $c$ axis expands by over $100 \%$. This expansion is consistent with our previously proposed 'spaghetti model', ${ }^{9}$ which requires shearing of $\mathrm{CP}$ chains along the crystallographic $c$-axis, and the intertwining of these same chains along the $a$ and $b$ axes. The unique structural behaviour of obtusely and acutely bent (1) is consistent with our previously reported mechanical and spectroscopic studies of this material. ${ }^{9}$ Hence, our high pressure studies suggest that the atomistic response of (1) to three-point bending does not follow directly from its high-pressure response. Although relatively low pressures appear to be generated initially, the significant bending results not from continued compression of the material, but from overall failure of the bulk lattice.

In summary, we report here the first high-pressure study of a known mechanically flexible coordination polymer, $\left[\mathrm{Zn}(\mu-\mathrm{Cl})_{2}(3,5 \text {-dichloropyridine })_{2}\right]_{\mathrm{n}},(\mathbf{1})$. Despite significant crystallographic anisotropy, single crystal X-ray diffraction revealed that all three crystallographic axes undergo identical relative compression. Moreover, the quasi-hydrostatic compression of (1) to $9.35 \mathrm{GPa}$ is completely reversible. Upon decompression, the unit-cell parameters of (1) relax with no detectable hysteresis. This reversibility is remarkable considering the exceptional plastic response of (1) to threepoint bending. Both X-ray diffraction and microfocus Raman spectroscopy suggest the onset of a structural phase transition between 4-5 GPa. This phase transition corresponds to distortion of the dichloro-pyridine ligands and results in loss of crystallographic symmetry. Symmetry mode analysis and DFT-D simulations confirm the critical role of a low-frequency vibrational mode in driving this phase transition by softening at increased pressure. This soft mode is polarised along the crystallographic $c$-axis, and hence compression of the $a$ and $b$ axes drives the phase transition. However, as established by previous Raman spectra, there is no distortion of the CP band during three-point bending. We therefore demonstrate how the atomistic response of a mechanically flexible coordination polymer to three-point bending differs significantly from its response to quasi-hydrostatic compression. Small increases in pressure certainly develop locally in the initial stages of mechanical bending and may therefore be amenable to studies through low high-pressure investigations, for example in elastically flexible materials. However, the large plastic flexibility exhibited by single crystals of (1) results not from continued compression, but rather by failure of the lattice, e.g. through slighting and intertwining of the $\mathrm{CP}$ chains. We suspect this to be a general mechanism for plasticity in single crystal coordination polymers.

\section{Experimental Section}

High-quality single crystals of $\left[\mathrm{Zn}(\mu-\mathrm{Cl})_{2}\left(3,5-\mathrm{Cl}_{2} \mathrm{Py}\right)_{2}\right]_{n}(\mathbf{1})$ were prepared by stirring reaction of zinc chloride with 3,5-dichloropyridine in ethanol and followed by slow evaporation from the same solvent within 7 days. A suitable crystal was loaded into a Merrill-Bassett diamond anvil cell (DAC) with 1:1 pentane:isopentane as pressure transmitting medium. Ruby spheres were used for pressure calibration. Single crystal X-ray diffraction was collected on a Bruker D8 Venture X-ray diffractometer using Mo- $\mathrm{K}_{\alpha}$ radiation at room temperature condition ( $c$ a $297 \mathrm{~K}$ ). Full details are given in ESI S1. High 
pressure Raman spectra were collected on using a powdered sample loaded in a Diacell One20DAC with 1:1 pentane:isopentane as pressure transmitting medium. Ruby fluorescence was used for pressure calibration. An excitation wavelength of $532 \mathrm{~nm}$ was used for spectroscopic measurements. DFT calculations were performed within the CASTEP v19.11 suite using the exchange correlation functional of Perdew-Burke-Ernzerhof (PBE) with the dispersion correction of Tkachenko and Scheffler (TS). Electron-nuclear interactions were modelled using norm-conserving pseudopotentials generated 'onthe-fly' within the CASTEP code. Phonon calculations were performed at the Brillouin zone centre by linear response methods. Full details in ESI S2.

\section{Acknowledgements}

The authors are grateful to Zedat (Freie Universität, Berlin) and BAM IT for computational resources. Thanks to Prof Simon Parsons (University of Edinburgh) for his assistance with single crystal data collection. Dr Ana Belenguer (University of Cambridge) is gratefully acknowledged for critically reading the manuscript. The authors are grateful to Prof Beate Paulus (Freie Universität Berlin) for ongoing support and many fruitful discussions.

\section{References}

1. Ahmed, E., Karothu, D. P. \& Naumov, P. Crystal Adaptronics: Mechanically Reconfigurable Elastic and Superelastic Molecular Crystals. Angew. Chem. Int. Ed. 57, 8837-8846 (2018).

2. Naumov, P. et al. The Rise of the Dynamic Crystals. J. Am. Chem. Soc. 142, 13256-13272 (2020).

3. Annadhasan, M. et al. Mechanophotonics: Flexible Single-Crystal Organic Waveguides and Circuits. Angew. Chem. Int. Ed. 59, 13852-13858 (2020).

4. Kenny, E. P., Jacko, A. C. \& Powell, B. J. Mechanomagnetics in Elastic Crystals: Insights from [Cu(acac) 2 ]. Angew. Chem. Int. Ed. 58, 15082-15088 (2019).

5. Kwon, T., Koo, J. Y. \& Choi, H. C. Highly Conducting and Flexible Radical Crystals. Angew. Chem. Int. Ed. 132, 16578-16581 (2020).

6. Owczarek, M. et al. Flexible ferroelectric organic crystals. Nat. Commun. 7, 13108 (2016).

7. Liu, H. et al. A Flexible Organic Single Crystal with Plastic-Twisting and Elastic-Bending Capabilities and Polarization-Rotation Function. Angew. Chem. 132, 13044-13050 (2020). 
8. Reddy, C. M. et al. Mechanical bending induced fluorescence enhancement in plastically flexible crystals of a GFP chromophore analogue. Angew. Chem. ange.202007760 (2020) doi:10.1002/ange.202007760.

9. Bhattacharya, B. et al. A Mechanistic Perspective on Plastically Flexible Coordination Polymers. Angew. Chem. Int. Ed. 59, 5557-5561 (2020).

10. Worthy, A. et al. Atomic resolution of structural changes in elastic crystals of copper(II) acetylacetonate. Nat. Chem. 10, 65-69 (2018).

11. Panda, M. K. et al. Spatially resolved analysis of short-range structure perturbations in a plastically bent molecular crystal. Nat. Chem. 7, 65-72 (2015).

12. Hayashi, S. \& Koizumi, T. Elastic Organic Crystals of a Fluorescent $\pi$-Conjugated Molecule. Angew. Chem. Int. Ed. 55, 2701-2704 (2016).

13. Đaković, M. et al. Mechanically Responsive Crystalline Coordination Polymers with Controllable Elasticity. Angew. Chem. Int. Ed. 57, 14801-14805 (2018).

14. Mondal, A. et al. Metal-like Ductility in Organic Plastic Crystals: Role of Molecular Shape and Dihydrogen Bonding Interactions in Aminoboranes. Angew. Chem. Int. Ed. 59, 10971-10980 (2020).

15. Dey, S. et al. Mechanically interlocked architecture aids an ultra-stiff and ultra-hard elastically bendable cocrystal. Nat. Commun. 10, 3711 (2019).

16. Commins, P., Karothu, D. P. \& Naumov, P. Is a Bent Crystal Still a Single Crystal? Angew. Chem. Int. Ed. 58, 10052-10060 (2019).

17. Rather, S. A. \& Saha, B. K. Thermal Expansion Study as a Tool to Understand the Bending Mechanism in a Crystal. Cryst. Growth Des. 18, 2712-2716 (2018).

18. Brock, A. J. et al. Elastically Flexible Crystals have Disparate Mechanisms of Molecular Movement Induced by Strain and Heat. Angew. Chem. Int. Ed. 57, 11325-11328 (2018). 
19. Hayashi, S. et al. Anisotropic Poisson Effect and Deformation-Induced Fluorescence Change of Elastic 9,10-Dibromoanthrathene Single Crystals. Angew. Chem. Int. Ed. 59, 16195-16201 (2020).

20. Zakharov, B. A. \& Boldyreva, E. V. High pressure: a complementary tool for probing solid-state processes. CrystEngComm 21, 10-22 (2019).

21. Cliffe, M. J. \& Goodwin, A. L. PASCal: a principal axis strain calculator for thermal expansion and compressibility determination. J. Appl. Crystallogr. 45, 1321-1329 (2012). 